\title{
Epidemiology of Irritable Bowel Syndrome in Albania
}

\author{
Adelina N. Galica ${ }^{1,2}$, Reitano Galica ${ }^{3}$, Dan L. Dumitrascu ${ }^{1}$
}

1) $2^{\text {nd }}$ Department of Internal Medicine, Iuliu Hatieganu University of Medicine and Pharmacy Cluj-Napoca, Romania

2) Department of Nursing, Faculty of Natural and Human Sciences, Fan S. Noli University, Korçe, Albania 3) Regional Hospital Korçe, Albania

Address for correspondence: Adelina Nicoleta Galica Department of Nursing, Faculty of Natural and Human Sciences, Fan S. Noli University, Korçe, Albania adelinagalica2016@gmail.com

Received: 11.06.2021

Accepted: 06.08.2021

\begin{abstract}
Background \& Aims: Irritable bowel syndrome (IBS) is a chronic condition, with a relatively high prevalence and represents a burden for the healthcare budgets. Knowing the prevalence of IBS is important for scientific and practical estimation of its impact. The recent Rome IV global study has shed light on the prevalence of IBS in many countries, but reports are lacking from other countries. The aim of this study was to assess the prevalence of IBS in Albania, a country from which we do not have relevant information.

Method: A prospective study was carried out in order to find out the prevalence of IBS in Albania. The target population was a sample of at least 500 Albanian adults (from a population of 2.2 million adults in this country. The questionnaires were distributed to the general population through three University centers from different areas of the country, through family doctors, chosen randomly, and by door to door by volunteer medical students. The questionnaires were self-completed by the participants and included 29 items: biographical data, IBS symptoms based on the Rome IV criteria, the symptoms that accompany IBS and the quality of life (QoL). The prevalence of IBS in the studied population, the sex ratio, the subtypes of IBS and the prevalence of different symptoms were calculated.

Results: We sent out 550 questionnaires and we received answers from 502 subjects (response rate $91 \%, 312$ females, median age: 42 years). IBS was present in 43 cases (8.6\%), without gender predominance. The IBS subtypes had the following values: IBS-C 58\%, IBS-D $28 \%$ and IBS-M $14 \%$. Women had a relatively higher risk for subtype IBS predominant constipation ( $\mathrm{OR}=1.13,95 \% \mathrm{CI}: 0.32-3.95, \mathrm{p}=0.84)$ but men appeared to be more prone to IBS predominant diarrhea $(\mathrm{OR}=1.3,95 \% \mathrm{CI}: 0.33-5.08, \mathrm{p}=0.7)$. The most affected age was over 50 years and the least affected was between $18-30$ years. Bloating was present in $81.4 \%$ of IBS respondents. The pain significantly influenced the daily activity in IBS: OR=12.9:1 (95\% CI: 6.18-27.31, p<0.0001). Education seems to be an important risk factor; people with only middle school education or less had an OR=4.5 (95\% CI: 2-10.54, $\mathrm{p}=0.0003$ ) of developing IBS than people with a higher education.

Conclusions: These are the first reported data on IBS in Albania. IBS in Albania is of 8.6\%, more than the average data in the Rome IV global study, but in the range obtained from other studies. The most common type is IBS-C; there is no gender predominance; it is more common in less educated persons; the influence of pain on daily activity is higher in IBS.
\end{abstract}

Key words: Albania - epidemiology - IBS - prevalence.

Abbreviations: DGBI: disorders of gut-brain interaction; FGID: functional gastrointestinal disorders; IBS: irritable bowel syndrome; IBS-C: IBS predominant constipation; IBS-D: IBS predominant diarrhea; IBS-M: IBS with mixed bowel habits; QoL: quality of life.

\section{INTRODUCTION}

Irritable bowel syndrome (IBS) is one of the most common digestive disorders of the gutbrain interaction and its standard diagnosis relies on the Rome IV criteria $[1,2]$.

Knowing the true prevalence of IBS worldwide has been a challenging quest. A systematic review form 2012 conducted by Lovell and Ford [3] considered studies from many countries but with different diagnostic criteria, finding a global pooled prevalence of IBS of $11.2 \%$, with wide variations among countries. In a systematic review from 2015, Rusu and Dumitrașcu [4] evaluated the data on IBS available from East European and former communist countries and found few data and high prevalence: $14 \%$ in Romania and $28 \%$ in Croatia.

The Rome Foundation Global Study [5] was conducted in 33 countries and concluded that most of the countries had IBS prevalence between $3 \%$ and $5 \%$. A prevalence of $5-10 \%$ is 
detected in studies in the United States, Europe, New Zealand, and Australia [6].

The prevalence of IBS is higher in younger aged and middle-aged adults [3,7]. The prevalence of IBS is higher in women [4-8] but this feature is not consistent everywhere; in USA [5] the prevalence was not significantly higher in women. A systematic review from 2020 on South Asian countries and India also do not find any gender predominance [9].

Patients with IBS are classified into the 4 subtypes, according to the Bristol Stool Form Scale, with the predominance of constipation (IBS-C), of diarrhea (IBS-D), mixed (IBS-M) or unspecified (IBS-U) [10]. Each subtypes IBS-C, IBS-D, IBS-M count about $1 / 3$ of the IBS cases [11]. Previous data on IBS subtypes showed that either IBS-D was most frequent [12] or IBS-C [13]. Bloating is a common symptom in the general population $(30 \%)[15,16]$ and is also present in $60-90 \%$ of patients with IBS, but bloating is a secondary element in the description of IBS [17].

Significant changes in quality of life were found in IBS compared with the general population [18]. It was concluded that approximately $75 \%$ of patients with IBS have depression and anxiety [19], while other studies have shown that a large number of women with IBS have gone through a trauma at some point in their lives [20].

Some countries do not have epidemiological data on IBS. One of them is Albania. In 2019, a small preliminary study (50 female participants) conducted in Albania found a 10\% prevalence, applying the Rome IV criteria [14].

The aim of our study was to provide more reliable epidemiological data on IBS in Albania.

\section{METHODS}

This is an epidemiological study conducted in Albania, during 2019-2020 and aiming to find the prevalence of IBS in the general population of this country.

\section{Protocol}

Albania has a population of 2.8 million inhabitants, from which 2.2 million have over 18 years. The target population was represented by more than 500 Albanian adults. A sample of adult individuals from the general population was asked to fill an ad-hoc structured questionnaire. The questionnaires were distributed through three university medical centers, through family doctors and door to door by volunteer medical students. The participating universities are located in three counties of Albania, one in South-East (Korçe), one in the center of the country (Tirana) and one in the North (Shkoder) (Fig. 1). All of those counties have universities that gather a variety of students and staff from both urban and rural areas, and we included medical staff and students who gave consent and completed the questionnaire. The family doctors were in the South-East County and offered the questionnaire to every subject from their files who presented at the office within a period of two weeks for non-organic gastrointestinal conditions or for mild nondigestive conditions, considering here the limitation caused by the pandemic in 2020 .

The subjects were informed about the investigated problem (IBS) and its importance and were assured of

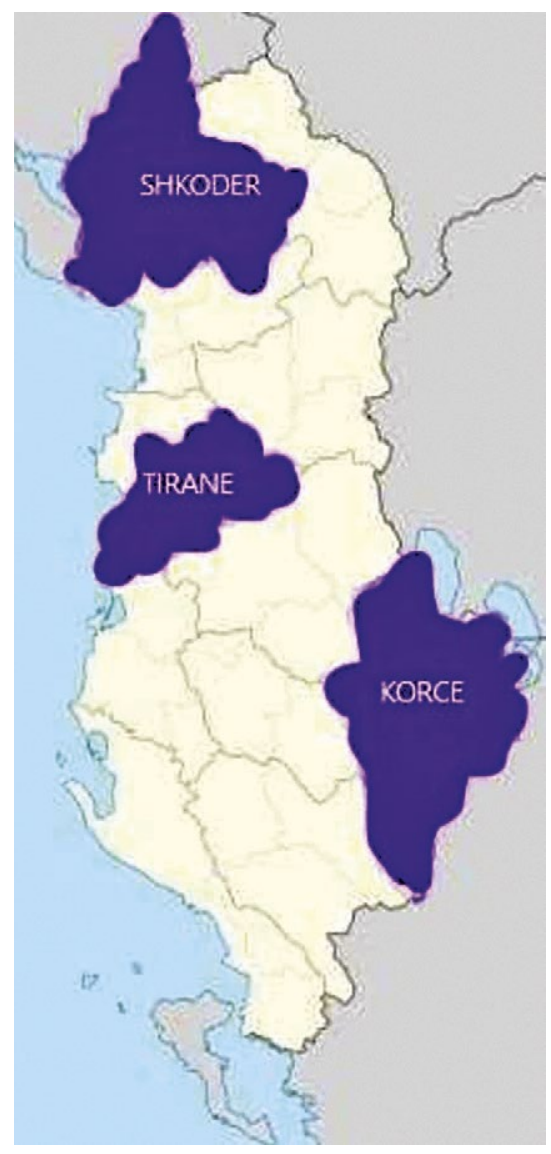

Fig. 1. Administrative map of Albania. The three counties where the study was carried out.

anonymity because no identification data were collected. The questionnaires were processed by applying the Rome IV criteria for diagnosing IBS.

\section{Questionnaire}

The data were collected through a questionnaire including questions on usual biographical data, medical history, IBS symptoms, symptoms that accompany IBS (bloating) and on the quality of life (Suppl. File). The questionnaire contained 29 items and required 10 minutes to be answered. Questionnaires were administered by instructed medical staff and filled by participants. Responding to questionnaires meant acceptance of protocol.

\section{Participants}

The questionnaire was administered to 550 subjects of both sexes, over 18 years. The inclusion criteria were: adults willing to take part in this survey. The exclusion criteria were: known gastrointestinal organic comorbidities. We included in this survey both medical staff and medical students, as individuals from the general population.

\section{Statistics}

The statistical program used for performing analysis was SPSS Version 20.0 for Windows. To evaluate the association between IBS and BMI, quality of life and demographic characteristics was used Chi-square test, with a values o $\mathrm{p}<0.05$ 
considered with statistical significance. Descriptive statistics was used to obtain the rates of demographic subgroups and subtypes of the condition. Odds ratio (OR) with 95\% confidence interval (CI) were calculated in order to compare and to describe relative risk of IBS and socio-demographic variables. A statistician was consulted for data analysis.

\section{Ethical Issues}

The study was approved by the local Ethical Committee and conducted according to the Helsinki Declaration on Human and Animal Studies.

\section{RESULTS}

We sent out 550 questionnaires and received 502 filled questionnaires, giving us a response rate of $91 \%$. All returned questionnaires were appropriately filled and were $100 \%$ acceptable for interpretation. The questionnaires distribution was as follows: $32 \%$ through universities, $32 \%$ through family doctors, $30 \%$ were distributed door-to-door and $6 \%$ were completed by medical staff.

Of the 502 respondents, 312 were females $(\mathrm{F} / \mathrm{M}=1.64)$. The age of the subjects studied was between 18 and 88 years (median $=42$ years, standard deviation $=17.62$ ). After applying the Rome IV criteria for IBS and the exclusion criteria, there were 43 (27 females and 16 males, F:M=1.68, with ages between 18 and 88 , median age: 60 , SD 17.25) subjects who met the diagnostic criteria for IBS, meaning a prevalence of $8.6 \%$.

We compared the prevalence by gender using OR with $95 \%$ $\mathrm{CI}$ and we observed that there is no gender-related risk for IBS (OR=1.03 95\%CI: 0.54-1.97). The IBS distribution according to age is displayed in Fig. 2. Seventy percentage of the studied population comes from urban areas and 30\% comes from rural areas. IBS subjects have the following distribution by living area: $74 \%$ come from urban areas and $26 \%$ come from rural areas. The probability of an urban area living person to have IBS is 1.26 times higher compared to a rural area living person (95\%CI: 0.62-2.58).

We looked for an association between the education level and IBS and we found that subjects with an elementary education have a 4.5 times higher risk of IBS (95\%CI: 2-10.54, $\mathrm{p}=0.0003$ ) than those in higher education (high school and upper).

Depending on the dominant symptom, we further calculated the prevalence of each IBS subtype and obtained the following results: IBS-C 58\%, IBS-D 28\% and IBS-M 14\%.

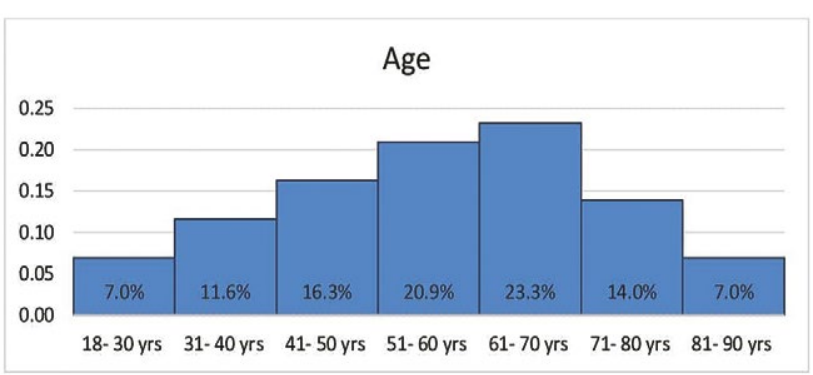

Fig. 2. Age distribution of IBS patients.
The prevalence of IBS-C in women was $59.2 \%$ and in men is $56.3 \%$. The OR for IBS-C in women compared to men is 1.13 (95\% CI: $0.32-3.95, \mathrm{p}=0.84$ ). The prevalence of IBS-D in the female group is $26 \%$ and the prevalence of IBS-D in the male group is $31.2 \%$. The OR for IBS-D in men compared to women is 1.3 (95\% CI: $0.33-5.08, p=0.7$ ). Subtype IBS-M is present at $14.8 \%$ of the females and at $12.5 \%$ from males and both sexes have the same odds to have IBS-M (OR 1.04, 95\%CI: 0.17-6.54 $\mathrm{p}=0.96$ ).

In the group of people diagnosed with IBS, 6 people did not provide the data needed to calculate BMI. Of those who provided data, $67.5 \%$ were overweight and obese. Statistical analysis with Chi-square test did not find a correlation between IBS and BMI $(\mathrm{p}=0.07)$.

The presence of IBS-associated symptoms was investigated both in the general population and specifically in the group of IBS-positive people and the results are as follows: 132 subjects stated the presence of bloating (26\%) and 35 IBS-positive subjects $(81.4 \%)$ have confirmed bloating at least one day a week in the last 3 months. Of these, more than half ( 24 out of 35) were women.

We assessed the constipation symptoms in the studied group; $113(22.5 \%)$ subjects experienced types 1 or 2 stool on the Bristol scale and 106 stated that they had a stool less than 3 times a week (with a frequency equal to or greater than $25 \%$ of defecation cases). Diarrhea was present in $15 \%$ of the general population: 78 individuals stated that in the last 3 months they had type 6 or 7 stools on the Bristol scale. Of these 78 , seven individuals (9\%) were previously diagnosed during life with IBS.

The analysis of the comorbidities in IBS patients revealed that $9.3 \%$ (4) of the patients had gastroesophageal reflux, $40 \%$ (17) had a diagnosis of functional constipation, $11.6 \%$ (5) had diabetes, $2.3 \%$ (1) had fibromyalgia and 2.3\% (1) had functional dyspepsia.

A colonoscopy was performed at $14 \%$ (6 out 43 ) of the IBS patients and at $4.3 \%$ (20 out of 459 ) of the non-IBS subjects.

The connection with stress is confirmed by $32 \%$ of the IBS patients that consider that their symptoms and stress are related, with a $\mathrm{RR}=1.75$ (95\%CI: 0.96-3.19, $\mathrm{p}=0.06$ ).

The subjective assessment of the QoL had these results among IBS patients: 34\% had poor QoL, 23\% had satisfactory QoL, 34\% believed they had a good QoL and only 7\% answered that had a very good QoL. The pain influences the daily activity in IBS; self-assessment of the QoL explained significant interference of pain (IBS vs non-IBS, OR=12.9, 95\% CI: 6.1827.31, $\mathrm{p}<0.0001)$.

\section{DISCUSSION}

We investigated a sample of 502 Albanian adults from a general population of 2.8 million including 2.2 million adults. This gives a representation of $0.02 \%$ from the general population. This percent is relevant, considering that the Global Study of Rome Foundation used samples of 2,500 individuals for countries with a larger population [7].

The prevalence of IBS in the studied population is $8.6 \%$; this value is higher than latest data but still in range of some studies $[3,5,6]$. In the population sample studied, the number 
of females was higher than males there is no sex predominance for IBS.

The lowest prevalence was in the age category $18-30$ years where only $2 \%$ of subjects were IBS positive ( 3 out of 151 ), compared to the age group 50+ where IBS patients represented $16 \%$ from this subgroup (28 out of 170 ). The results here are in contrast with the results of other studies that have shown that IBS was more common in young people than in the elderly $[7,21]$.

To test the hypothesis of a higher frequency of IBS in people in urban areas, we compared the data and observed that IBS in Albania was more common in urban areas than in rural areas.

In respect to IBS subtypes, we found that the IBS-C subtype was the most common, followed by the IBS-D subtype and then by the IBS-M, as reported in the literature [7, 13], but a new research in a Bulgarian study found out that IBS-M was the predominant subtype in their country [21]. Invasive investigation such as a colonoscopy were more frequently performed at IBS patients than in the rest of the subjects in study.

Bloating was a more common symptom in people with IBS than in the general population (81.4\% compared to $26 \%)$ and women were more affected by this aspect than men. Symptoms of constipation were present in approximately $1 / 5$ of the studied population and liquid stools were present in $15.5 \%$ of the general population.

Subjective assessment of health is an important aspect of the quality of life of patients with IBS and the study reveals that people with IBS rate their health as satisfactory or poor in more than half of cases.

A group of $32 \%$ of the IBS patients admitted that stress and their symptoms are highly related.

The study has several strengths: it is a representative sample of the number of inhabitants in Albania; it includes a large segment of age and people with different occupations from different regions of the country. The weak point of the study is that it should have a wider distribution over the territory of the country.

\section{CONCLUSIONS}

IBS prevalence in Albania is $8.6 \%$, without gender predominance. This is the first study that estimates the prevalence of IBS in an Albanian adult population. The results obtained from the study are an important stimulus for further research of the functional gastrointestinal disorders in this area and meanwhile cover at least partially the white spot on SouthEastern European countries' map with data on IBS.

\section{Conflicts of interest: None to declare.}

Authors' contribution: A.G. developed the questionnaire, selected the participants, analyzed data and wrote the draft. R.G. assisted A.G. in carrying out the study by contributing to questionnaire distribution, collection and analysis. D.L.D. conceived the study, mentored its progress, contributed to the writing of the manuscript. All authors read and agreed the final version.

Acknowledgements: Paola Vasili, Maria Dojce, Bendis Kraja and all the volunteer students are thanked for their contribution in distributing the questionnaires in the territory.

\section{REFERENCES}

1. Drossman DA. Functional gastrointestinal disorder and the Rome IV process. In: Drossman DA, Chang L, Chey WD, et al. (Eds.). Functional gastrointestinal disorders. Disorders of brain-gut interaction. Volume 1. 4th ed. Raleigh, NC: Rome Foundation, 2016:1-32.

2. Schmulson MJ, Drossman DA. What is new in Rome IV. J Neurogastroenterol Motil 2017;23:151-163. doi:10.5056/jnm16214

3. Lovell RM, Ford AC. Global prevalence of and risk factors for irritable bowel syndrome: a meta-analysis. Clin Gastroenterol Hepatol 2012;10:712-721.e4. doi:10.1016/j.cgh.2012.02.029

4. Rusu F, Dumitrascu DL. Epidemiology of irritable bowel syndrome in the former communist countries from Eastern Europe: a systematic review. Clujul Med 2015;88:146-151. doi:10.15386/cjmed-449

5. Palsson OS, Whitehead W, Tornblom H, Sperber AD, Simren M. Prevalence of Rome IV functional bowel disorders among adults in the United States, Canada and the United Kingdom. Gastroenterology 2020;158:1262-1273.e3. doi:10.1053/j.gastro.2019.12.021

6. Sperber AD, Dumitrascu DL, Fukudo S, et al. The global prevalence of IBS in adults remains elusive due to the heterogeneity of studies: A Rome Foundation working team literature review. Gut 2017;66:10751082. doi:10.1136/gutjnl-2015-311240

7. Sperber AD, Bangdiwala SI, Drossman DA, et al. Worldwide Prevalence and Burden of Functional Gastrointestinal Disorders, Results of Rome Foundation Global Study. Gastroenterology 2021;160:99-114.e3. doi:10.1053/j.gastro.2020.04.014

8. Lovell RM, Ford AC. Effect of gender on prevalence of irritable bowel syndrome in the community: systematic review and meta-analysis. Am J Gastroenterol 2012;107:991-1000. doi:10.1038/ajg.2012.131

9. Black CJ, Ford AC. Global burden of irritable bowel syndrome: trends, predictions and risk factors. Nat Rev Gastroenterol Hepatol 2020;17:473-486. doi:10.1038/s41575-020-0286-8

10 Lewis SJ, Heaton KW. Stool form scale as a useful guide to intestinal transit time. Scand J Gastroenterol 1997;32:920-924. doi:10.3109/00365529709011203

11. Enck P, Aziz Q, Barbara G, et al. Irritable bowel syndrome. Nat Rev Dis Primers 2016;2:16014. doi:10.1038/nrdp.2016.14

12. Oka P, Parr H, Barberio B, Black CJ, Savarino EV, Ford AC. Global prevalence of irritable bowel syndrome according to Rome III or IV criteria: a systematic review and meta-analysis. Lancet Gastroenterol Hepatol 2020;5:908-917. doi:10.1016/S2468-1253(20)30217-X

13. Ford AC, Lacy BE, Talley NJ. Irritable Bowel Syndrome. N Engl J Med 2017;376:2566-2578. doi:10.1056/NEJMra1607547

14. Dumitrascu DL, Babin A, Bordin DS, et al. Recent Data on Irritable Bowel Syndrome from some Central and East European Countries. J Gastrointestin Liver Dis 2020;29:247-250. doi:10.15403/jgld-2407

15. Chang L, Lee OY, Naliboff B, Schmulson M, Mayer EA. Sensation of bloating and visible abdominal distension in patients with irritable bowel syndrome. Am J Gastroenterol 2001;96:3341-3347. doi:10.1111/ j.1572-0241.2001.05336.x

16. Sandler RS, Stewart WF, Liberman JN, Ricci JA, Zorich NL. Abdominal pain, bloating, and diarrhea in the United States: prevalence and impact. Dig Dis Sci 2000;45:1166-1171. doi:10.1023/a:1005554103531

17. Pop LL, Mureșan IA, Dumitrașcu DL. How much bloating in the irritable bowel syndrome? Rom J Intern Med 2018;56:221-226. doi:10.2478/ rjim-2018-0017

18. Gralnek IM, Hays RD, Kilbourne A, Naliboff B, Mayer EA. The impact of irritable bowel syndrome on health-related quality of life. Gastroenterology 2000;119:654-660. doi:10.1053/gast.2000.16484 
19. Holtmann GJ, Ford AC, Talley NJ. Pathophysiology of irritable bowel syndrome. Lancet Gastroenterol Hepatol 2016;1:133-146. doi:10.1016/ S2468-1253(16)30023-1

20. Defrees DN, Bailey J. Irritable Bowel Syndrome: Epidemiology, Pathophysiology, Diagnosis, and Treatment. Prim Care 2017;44:655671. doi:10.1016/j.pop.2017.07.009
21. Nakov R, Dimitrova-Yurukova D, Snegarova V, et al. Prevalence of Irritable Bowel Syndrome, Functional Dyspepsia and their Overlap in Bulgaria: a Population-Based Study. J Gastrointestin Liver Dis 2020;29:329-338. doi:10.15403/jgld-2645 